\title{
Contraptions for intrauterine contraception
}

\author{
Lindsay Edouard
}

\section{Summary}

Intrauterine contraception is underutilised largely due to its reputed association with infections. The Copper T-380A, one of the most cost-effective methods of contraception and the most widely used intrauterine contraceptive device in the world, is effective for at least 12 years and is also used for emergency contraception. The levonorgestrelreleasing intrauterine system (LNG IUS) is extremely useful for treating menorrhagia.

A renaissance of intrauterine contraception is overdue and will necessitate community-wide information campaigns to stimulate demand generation, implementation of service guidelines that avoid restrictive eligibility criteria, and access to service providers with special training in counselling and clinical skills.

\section{Purveyor policies}

Looking back 25 years to 1983 , the intrauterine contraceptive device (IUD) was very much in the forefront of discussions on contraception, ${ }^{1}$ including its value for emergency contraception. When a district circular barred the provision of all emergency contraception in the local clinics, a doctor sought advice as he felt that "this edict is a great interference with my clinical freedom". Whereas health authorities were considered to be "within their rights to issue such an edict", its contents were contrary to a parliamentary statement of the Minister of Health and the doctor was told to be "at liberty to prescribe privately any form of treatment you consider advisable". 2

Emergency contraception was not promoted optimally despite detailed guidelines from the Clinical and Scientific Advisory Committee of the National Association of Family Planning Doctors. The value both of hormonal pills and IUDs for emergency contraception had been recognised only after the initial marketing of those products for nonemergency contraception and pharmaceutical companies were reluctant to go through the bureaucratic application procedures and expense of registration of the additional indication with licensing authorities. In the absence of a product licence for emergency contraception, doctors could prescribe the product but representatives of pharmaceutical companies could neither discuss that indication nor provide related educational materials. ${ }^{3}$

The health service reorganisation of April 1982, with its move away from well-women services to their incorporation within community health with its emphasis on child health, threatened family planning. It was suggested that a closer relationship with child health would enable family planning doctors to provide a true family service with counselling being prominent. 4,5

\section{Provider perspectives}

With the increasing popularity of general practice for a career and the inclusion of family planning in its vocational training programmes, family planning services were expected to become increasingly integrated within that

J Fam Plann Reprod Health Care 2008; 34(3): 199-201

Algiers, Algeria

Lindsay Edouard, FRCOG, FFSRH, Member of the Editorial Advisory Board

Correspondence to: Professor Lindsay Edouard. E-mail: soranae@gmail.com discipline. Concern was expressed that the limited workload for training in IUD insertion was being misused by doctors who were seeking mere certification with no actual intention of fitting IUDs in their future practice. It was felt that IUD insertion training should be reserved for those committed to the subsequent provision of IUD services, with specialist family planning clinics being proposed for referrals from general services. 4,5 The revised guidelines for training recognised the need for flexibility to suit both local circumstances and the 'trinity' of settings: hospital, general practice and community clinics. It was hoped that the guidelines could also be useful for initiating discussions in other countries. ${ }^{6}$

David Bromham, a visionary who became the first chairman of the Faculty of Family Planning and Reproductive Health Care in 1993, clearly stated the primordial importance of practical skills and counselling enthusiasm of the IUD inserter for increasing acceptability of the method. ${ }^{7}$ With individual preferences for carrying out clinical procedures, there were major differences in techniques for IUD insertion and removal following lost threads. With IUD insertion being easier with countertraction on the cervix, gynaecologists tended to promote instruments such as the volsellum or tenaculum: as they can cause pain and bleeding, it was felt that those instruments should be used only in exceptional circumstances. The use of the gentler Judd-Allis tissue forceps was advocated, with application well away from the external os to avoid any interference with the uterine sound and IUD introducer. 8

Removal of IUDs with missing threads posed special challenges and it seemed that the administration of glyceryl trinitrate relaxed the cervix, thereby facilitating the procedure and reducing pain. ${ }^{9}$ A randomised trial was initiated to compare the value of various retrievers for the removal of IUDs following lost threads and the investigators invited referrals for the prompt recruitment of subjects. 10

\section{Public perceptions}

Acceptability of IUDs is adversely affected by misconceptions pertaining to their mechanism of action, ${ }^{11}$ body disturbance and fertility impairment as well as cultural dogmas and religious customs, especially in relation to vaginal bleeding. ${ }^{12}$ With the substantial increase in use of IUDs in the preceding years, there was an accumulation of observational data on possible side effects and increasing suspicion of a causal relationship between IUD use and ectopic pregnancy. ${ }^{13}$ The addition of copper or a hormone, to address problems with inert IUDs, led to speculations of "problems relating to the effective life of the agent in the uterus" with their anticipated replacement every 2 or 3 years being "a nuisance to the woman and an extravagance". 1

Complications from IUD use accounted for a significant number of gynaecological admissions. ${ }^{14}$ Furthermore, there was a debate on the role of IUDs in the aetiology of pelvic inflammatory disease, with its longterm sequel of impaired fertility, but it was acknowledged that analyses suffered from the lack of an appropriate control group. ${ }^{1}$

\section{Paraphernalia}

Commodity security was of concern as private pharmacies did not always stock IUDs, whereas in public facilities 
there was the risk of misuse of resources through overstocking of IUDs that needed to be destroyed after their expiry date. With the numerous types of IUD on the market, there was much confusion regarding their utilisation and no satisfactory explanation for the widely different recommendations from manufacturers regarding shelf life: normally varying from 3 to 5 years, it was unlimited for one type. ${ }^{15}$

At a meeting held in October 1982, it was noted that practitioners were increasingly recommending that copperbearing IUDs be used for about 4 years despite their being licensed for 2 years only. ${ }^{16}$ But within a year, the Journal was informing its readers of the approved use of two copperbearing IUDs for 4 and 5 years, besides publishing an article comparing the cost-effectiveness of two copper-bearing IUDs needing replacing after 2 and 4 years, respectively. ${ }^{17}$

Progestasert ${ }^{\circledR}$, the progesterone-releasing device, was recognised as being valuable for those suffering from bleeding or pain with other IUDs, but with its short licensed duration of use, its perceived high cost and its demise from an association with ectopic pregnancies, there was much hope for the development of the LNG IUS. ${ }^{1}$

The IUD saga had started just before 1910 with the efforts of Richter with silkworm gut, which were quickly followed by the Graefenberg ring. From the late 1950s, there was a revival consisting mainly of barium-containing polyethylene models with the diversity being reflected in names such as the Birnberg bow, Delta-T device, Lippes loop, Latex leaf, Margulies spiral and Saf-T-Coil; designs also included other shapes such as the butterfly, heart, shamrock, shield, spring, triangle and wing.

The Dalkon Shield had twice the pregnancy failure rate but only one-third of the expulsion rates when compared to contemporaneous IUDs. It had a multifilament tail through which microorganisms could ascend into the expanding uterus as pregnancy progressed. With the American practice of not removing IUDs in cases of continuing pregnancy, the associated septic mid-trimester abortions caused a disaster in the USA from 1973. By the mid-1980s, IUDs had been withdrawn from the market in the USA as a result of commercial decisions by the manufacturers who were fearful of litigation for infection and infertility. ${ }^{18}$ However, in the rest of the world, IUDs continued to be used whether inert, like the Lippes loop and Saf-T-Coil, or medicated with either copper or progestogen. In China, an inert stainless steel ring was used extensively until 1993 when it started to be replaced by the more effective copperbearing IUDs. ${ }^{19}$ Frameless IUDs were designed to reduce bleeding and pain ${ }^{20}$ but their continuation rates were no better than conventional copper devices.

The oft-quoted practice of inserting small stones in the uteri of camels to prevent pregnancies during long caravan journeys has never been adequately documented. However, fetal bone fragments, which might be retained in the human uterus after an abortion, are known to produce a contraceptive effect. ${ }^{21,22}$

\section{Present position}

The safety of IUDs is well recognised: risks relate mainly to bleeding, infection and perforation. With the uterus being in close proximity to other organs in the pelvis, perforation can lead to adhesions between their surfaces. As IUDs are most effective at preventing intrauterine pregnancy, and rather less so for tubal and other pregnancies, the proportion (as opposed to rate per woman-year) of ectopic pregnancies is increased when conception occurs in the presence of an IUD. With contraceptive failure and a continuing intrauterine pregnancy, the IUD should be removed as soon as possible to reduce risk of miscarriage.
The unfortunate American experience with the Dalkon Shield led to misinformation on the risk of infection for IUDs, but as women in monogamous relationships were not found to be at increased risk, it was claimed that "men, not IUDs, cause pelvic infection". ${ }^{23}$ Pelvic infection does occasionally occur after insertion of an IUD, the risk being primarily in the subsequent 3 weeks ${ }^{24}$ and commonly related to the presence of either chlamydia or gonorrhoea: the subsequent incidence of infection is similar to that of non-users. Antibiotic cover is not recommended for insertion of an IUD unless facilities for screening prospective users deemed to be at a high risk are limited. Behavioural factors should be assessed rather than relying on information on the local prevalence of chlamydia and gonorrhoea. ${ }^{24}$ The confidence of IUD providers to manage sexually transmitted infections (STIs) is enhanced by the provision of integrated reproductive health services. Prevention of infection depends on three measures: screening for STIs prior to insertion, strict aseptic procedures for insertion and, most importantly, reducing the frequency of reinsertion by leaving the IUD in situ for its entire effective lifespan, unless removal is either clinically indicated or requested by the user. ${ }^{24}$ IUDs do not influence the risk of either acquiring or transmitting HIV, do not lead to increased morbidity in those with HIV, and can usually be used by HIV-positive individuals who are receiving clinical care. Women with AIDS should be taking effective antiretroviral therapy. ${ }^{24}$

IUDs with a copper surface area of at least $300 \mathrm{~mm}^{2}$ provide effective long-term contraception. The most widely tested device worldwide, the Copper T-380A, has $380 \mathrm{~mm}^{2}$ of copper on its plastic stem and arms. It provides contraception for at least 12 years, although its UK licence is for 10 years of use. It has similar effectiveness to female surgical sterilisation for a commodity cost in the international public sector of around US\$0.50. The copper IUD is one of the most cost-effective methods of contraception and is safe, cheap, easy to use and quickly reversible. Its most frequent use for interval insertion should be supplemented by recognising its value for insertion after pregnancy, whether postabortal or postpartum, as a convenient procedure to satisfy demand and to address unmet need when there is poor access to clinical services. Postabortal insertion can be performed in the absence of infection or other major complications, while postpartum insertion should be within 48 hours of delivery, specially during the 10 minutes following removal of the placenta, or alternatively after 4 weeks at a postnatal or well-baby visit. ${ }^{25}$

For emergency contraception, insertion of a copper IUD has the advantages of being much more effective than oral hormonal methods, being also indicated for use for 5 days after unprotected intercourse and in certain circumstances beyond that time, and providing additional long-term contraception when left in situ. However, IUD insertion for this purpose is greatly underutilised, probably due to provider bias, lack of practical training in clinical skills and because of the increasing availability of hormonal methods without the requirement for medical contact.

The LNG IUS (Mirena ${ }^{\circledR}$ ) provides contraception for at least 5 years and is an alternative to female sterilisation in terms of cost and effectiveness. Furthermore, it has the advantage of being reversible besides having the major non-contraceptive benefit of reduction in uterine bleeding, to the extent that it is recommended and licensed as a firstline treatment for menorrhagia. When seeking sterilisation, women find the LNG IUS to be a valuable alternative; it is imperative that this choice be offered during counselling. 26,27 


\section{Prospects}

With the global prevalence rate for all modern contraceptive methods being $56 \%$ among women who are either married or in a union, the highest method-specific rate is for female sterilisation at $20 \%$ with the second highest being for the IUD, which is the most popular reversible method at $16 \%$, but with wide geographical variations: $14 \%$ in Europe, as high as $45 \%$ in China and $44 \%$ in Cuba and less than $2 \%$ in the USA and sub-Saharan Africa. ${ }^{28}$ These geographical differences in the pattern of IUD utilisation provide testimony that the time is ripe for a renaissance of intrauterine contraception by ensuring its prominence in the range of methods for both limiting and spacing births. With the current trend for women to complete childbearing at a younger age, copper IUDs offer a most valuable alternative to permanent irreversible contraception in the international setting. ${ }^{29}$

IUD insertion is a simple non-surgical task that can be performed easily in a variety of settings by well-trained primary care providers such as nurses and midwives, as exemplified by current practice in numerous countries including Sweden and the USA. ${ }^{24}$ Despite extensive IUD training, a shortage of skilled inserters is often a problem. Trainees should be selected according to criteria that indicate their likely involvement in IUD activities during subsequent practice. When a high turnover in jobs is a problem, on-the-job competency-based training should be emphasised. Increasing the utilisation of IUDs will necessitate advocacy with policymakers and generation of demand through communication, with unbiased information and counselling for potential users. It is crucial to dispel the myths and misconceptions that are often rampant among non-users. With good provision of information by trusted advocates, side effects are better tolerated, thus increasing continuation rates. Up-to-date evidence and authoritative guidance from professional bodies, including the World Health Organization, should be used to develop local service guidelines on eligibility criteria and to eliminate inappropriate barriers due to restrictive policies. ${ }^{24}$ Wide dissemination and implementation of service guidelines, especially for the training of service providers, should be part of initiatives to reposition family planning.

Statements on funding and competing interests

Funding None identified.

Competing interests None identified.

References

1 McEwan J. Intrauterine contraception: what next and why? $\mathrm{Br}$ J Fam Plann 1983; 9: 3-11.

2 Biddell S. Questions and answers: post-coital contraception. $\mathrm{Br}$ J Fam Plann 1983; 8: 149.

3 Postcoital contraception [Editorial]. Br J Fam Plann 1983; 9: 41-42.

4 Pollitt Y. Reports - Affiliated Groups Meeting. Br J Fam Plann 1983; 9: 26-27.

5 Main A. Family planning training. Br J Fam Plann 1983; 8: 140-141.

6 Revised guidelines for family planning training [Editorial]. $\mathrm{Br} \mathrm{J}$ Fam Plann 1983; 9: 1.

7 Evans B. Reports - North West Society for the Study of Sexual Medicine and Family Planning. Br J Fam Plann 1983; 8: 143-144.

8 Watson C. Use of a tenaculum. Br J Fam Plann 1983; 9: 99.

9 McGarry JM, Ford RG. Transiderm-Nitro. Br J Fam Plann 1983; 9: 98.

10 Bounds W. Retrieval of lost IUD threads. Br J Fam Plann 1983; 8: $148-149$

11 Morris G. Ethical issues in reproductive medicine. $\mathrm{Br} J$ Fam Plann 1983; 9: 29-30.

12 Rashid J. Contraceptive use among Asian women. Br J Fam Plann 1983; 8: 132-135.

13 Woo JSK, Li DFH. Ovarian pregnancy and the intrauterine contraceptive device. Br J Fam Plann 1983; 9: 22-24.
14 Fraundorfer MR. The intra-uterine device and hospital admission. Br J Fam Plann 1983; 9: 79-84.

15 Anonymous. From the Clinical and Scientific Advisory Committee. Br J Fam Plann 1983; 9: 2.

16 Friedmann B. Ninth Fertility Control Symposium. Br J Fam Plann 1983; 8: 144-147.

17 Morfitt JM. Novagard versus Gravigard: a cost-effectiveness study. Br J Fam Plann 1983; 9: 59-62.

18 Sivin I. Another look at the Dalkon Shield: meta-analysis underscores its problems. Contraception 1993; 48: 1-12.

19 Bilian X. Chinese experience with intrauterine devices. Contraception 2007; 75(6 Suppl.): S31-S34.

20 O'Brien PA, Marfleet C. Frameless versus classical intrauterine device for contraception. Cochrane Database Syst Rev 2005; (1): CD003282.

21 Panama S, Triolo O, Arezio P. Prolonged retention of fetal bones: intrauterine device and extrauterine disease. Clin Exp Obstet Gynecol 1990; 17: 47-49.

22 Srofenyoh E, Addison M, Dortey B, Kuffour P. Intrauterine retained fetal bones as a cause of secondary infertility. Ghana Med J 2006; 40: 105-109.

23 Darney PD. Time to pardon the IUD? N Engl J Med 2001; 345: 608-610.

24 Population Reports. New Attention to the IUD: Expanding Women's Contraceptive Options to Meet their Needs (Series B, Number 7). Baltimore, MD: Johns Hopkins Bloomberg School of Public Health, 2006.

25 Grimes D, Schulz K, Van Vliet, H Stanwood N. Immediate postpartum insertion of intrauterine devices. Cochrane Database Syst Rev 2003; (1): CD003036.

26 National Institute for Health and Clinical Excellence (NICE). Long-Acting Reversible Contraception (NICE Clinical Guideline 30). London, UK: NICE, 2005

27 Mattinson A, Mansour D. Female sterilisation: is it what women really want or are alternative contraceptive methods acceptable? J Fam Plann Reprod Health Care 2006; 32: 181-183.

28 United Nations. World Contraceptive Use 2007. Publication ST/ESA/SER.A/273. New York, NY: Population Division, Department of Economic and Social Affairs, United Nations, 2008.

29 Sivin I. Utility and drawbacks of continuous use of a copper T IUD for 20 years. Contraception 2007; 75(6 Suppl.): S70-S75.

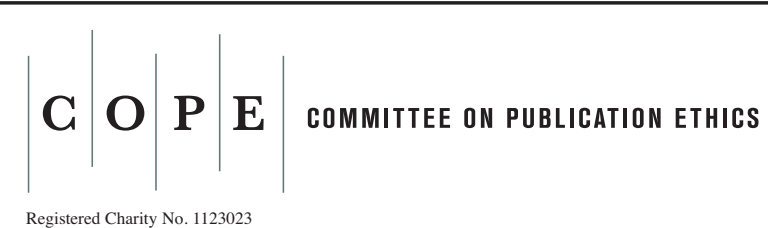 \\ EDITOR wanted for COPE's quarterly online 8-page newsletter for its 3000 members.}

We are looking for someone interested in leading the debate on publication ethics and seeking out news and features on this topic.

Administrative help will be given. Previous knowledge of web publishing not essential. An honorarium will be payable.

For further information and to discuss the nature of the newsletter, contact the COPE Chair: Dr Harvey Marcovitch.

E-mail: h.marcovitch@btinternet.com. Tel: 01295738100.

COPE, PO Box 39, Harleston IP20 9WR, UK Tel: 02073836602 www.publicationethics.org.uk 